\title{
Melatonin and jet lag
}

World class sportspersons must travel widely for international competitions and sports camps. As a result, they will suffer from circadian dyschronism, more popularly known as "jet lag" if they fly across several time zones to the east or west.

Normally, humans work in the daytime and sleep at night. This pattern is due not only to a behavioural response to the rhythmic environment, but also to a "body clock". The body clock is situated in the base of the brain (the hypothalamic suprachiasmatic nuclei) and exerts its effects throughout the body by producing daily rhythms in core temperature, plasma melatonin and adrenaline, and the sympathetic nervous system.

During the daytime, the body clock facilitates physical and mental activity by raising body temperature and plasma adrenaline, so harmonising with our behaviour and environment. In the evening these variables are lowered in preparation for sleep. At the same time, plasma melatonin levels rise and contribute to the increase in fatigue. ${ }^{23}$ In the second half of the night, the body clock is responsible for preparing the body for waking by reversing these changes.

The synchronisation between the environment and body clock is produced in several ways. Firstly, bright light directly stimulates an individual and suppresses melatonin secretion, ${ }^{4}$ so removing its hypnotic effect. Secondly, bright light and melatonin can independently adjust the phase of the body clock. Light signals falling on the retina between 0500 and 1100 hours, that is just after the temperature trough, advance the body clock, and those falling before the trough (2100-0300 hours) delay it. ${ }^{5}$ The size of the shift is related also to the logarithm of light intensity, domestic lighting producing changes of up to two hours. ${ }^{6}$ Melatonin ingestion in the morning produces a delay, and in the afternoon and early evening an advance, of the body $\operatorname{clock}^{78}$; however, the detailed times of ingestion of melatonin to produce the largest phase shifts are not as clearly known as for light.

The rhythmicity due to the body clock is of direct relevance to athletes. ${ }^{19}$ Muscle strength, adrenaline secretion, short term maximal power output, and self chosen work rate are all greatest at about 1800 hours; and at about this time also is the highest speed for mental performance involving reaction time and manual dexterity, and the perceived exertion for a given amount of exercise is least. Most personal best performances are set at this time.

Flight across more than three time zones produces a problem because the body clock adjusts its phase only slowly, about one or two hours per day under normal circumstances. Until adjustment has occurred, the normal matching between the body clock and the environment will be lacking. Individuals will initially feel tired during the new daytime (at a time coincident with night on the time zone just left) and yet unable to sleep well during the new night (which will coincide with day in the time zone just left). ${ }^{10}$ The loss of sleep will certainly affect mood and powers of concentration adversely. This, coupled with attempting activities at a time perceived by the body clock as being night, will result in poorer training schedules and even competition results. ${ }^{11}$

For competitors at the international level, most results indicate a poorer performance (of activities involving complex mental activity rather than physical strength), a feeling of lethargy, and a general loss of motivation. ${ }^{1213}$

In practice therefore advice to athletes concentrates on behaviour during travel itself, on training in the days immediately after the flight, and on methods to promote adjustment of the body clock. ${ }^{11}$ During the flight, dehydration and stiffness should be avoided (by taking copious amounts of water and fruit juice, and by short walks and stretching exercises), and the times of taking naps and meals should be determined by habits in the new, rather than the old, time zone. Training programmes should be devised with the consideration that some of the new daytime will correspond to night in the time zone just left and that the athlete or games player will also be suffering from the effects of sleep loss. Restricted training schedules and reduced levels of achievement should be expected by coach and athlete or player.

Two obvious countermeasures to jet lag exist. The first is to promote sleep and alertness at the appropriate times; the second is to promote adjustment of the body clock to the new time zone. In both of these, melatonin may have an important role. ${ }^{14}$

It is well established that ingestion in the evening in the new time zone reduces the symptoms of jet lag, particularly sleeping difficulties. ${ }^{15}$ However, as with other hypnotics, there is the need to ensure also that the quality of sleep is sufficient and that alertness and mental performance after sleep are not impaired by any residual effects of melatonin. The results of such studies have been contradictory ${ }^{16}{ }^{17}$; a resolution of the position is awaited.

The effectiveness of melatonin after time zone transitions may instead, or in addition, arise from promoting adjustment of the body clock. ${ }^{7814}$ Good field data testing this possibility do not yet exist. Moreover, some recent laboratory studies do not support an important role for melatonin in causing a shift of all rhythms (as would be the case if the body clock itself were shifted), ${ }^{18}$ particularly if shifts due to light are present also. ${ }^{19} 20$ The interaction between melatonin and light induced phase shifts requires further work.

In brief, the efficacy of melatonin in alleviating the fatigue associated with jet lag is not in dispute, but to what extent the beneficial effects of melatonin extend to promoting performance and mood in the new daytime, and an appropriate adjustment of the body clock, is less clear.

There are other doubts associated with the use of melatonin. It is not licensed in several areas of the world (including the United Kingdom), and the effects of those preparations that are commercially available, which contain other substances in addition to melatonin, have not been examined in athletes and games players. Moreover, in some individuals, there are some adverse side effects. The British Olympic Association has advised caution in the use of melatonin, particularly if individuals have not already established that it has beneficial effects without adverse side effects in their particular case. This advice seems the most reasonable at the present time.

J WATERHOUSE T REILLY G ATKINSON

Research Institute for Sport and Exercise Sciences,

Liverpool fohn Moores University

1 Reilly T, Atkinson G, Waterhouse J. Biological rhythms and exercise. Oxford: Oxford University Press, 1997.

2 Dawson D, Encel N. Melatonin and sleep in humans. F Pineal Res 1993;15: $1-12$.

3 Lavie P, Haimov I, Shochat T. Melatonin: shutting off the wakefulness system. Front Horm Res 1997;23:149-60.

4 Lewy A, Wehr T, Goodwin G, et al. Light suppresses melatonin secretion in humans. Science 1980;210:1267-9.

Minors D, Waterhouse J, Wirz-Justice A. A human phase-response curve to light. Neurosci Lett 1991;133:36-40. 
6 Boivin D, Duffy J, Kronauer R, Czeisler C. Dose-response relationship for resetting of human circadian clock by light. Nature 1996;379:540-2.

7 Lewy AJ, Ahmed S, Jackson JML, et al. Melatonin shifts human circadian rhythms according to a phase-response curve. Chronobiol Int 1992;9:38092 .

8 Krauchi K, Cajochen C, Mori D, et al. Early evening melatonin and S-20098 advance circadian phase and nocturnal regulation of core body temperature. Am f Physiol 1997;41:R1178-88.

9 Atkinson G, Reilly T. Circadian variation in sports performance. Sports Med 1996;21:292-312.

10 Ferrer CF, Bisson RD, French J. Circadian rhythm desynchronosis in military deployments: a review of current strategies. Aviat Space Environ Med 1995;66:571-8.

11 Reilly T, Atkinson G, Waterhouse J. Travel fatigue and jet-lag. I Sports Sci $1997 ; 15 \cdot 365-9$.

12 Smith RS, Guilleminault C, Efron B. Circadian rhythms and enhanced athletic performance in the National Football League. Sleep 1997;20:362-5.

13 Steenland K, Deddens JA. Effect of travel and rest on performance of professional basketball players. Sleep 1997;20:366-9.
14 Dawson D, Armstrong SM. Chronobiotics: drugs that shift rhythms. Pharmacol Ther 1996;69:15-36.

15 Arendt J, Aldhous M. Further evaluation of the treatment of jet-lag by melatonin: a double-blind crossover study. Annual Review of Chronophar macology 1988;5:53-6.

16 Atkinson G, Reilly T, Waterhouse J, et al. Pharmacology and the travelling athlete. In: Reilly T, Orme M, eds. A clinical pharmacology of sports and exercise. Amsterdam: Elsevier, 1997:293-301.

17 Kirby A, Adams B, Crowley J. Melatonin efficacy in aviation missions requiring rapid deployment and night operations. Aviat Space Environ Med 1996;67:520-4.

18 Middleton B, Arendt J, Stone BM. Complex effects of melatonin on human circadian rhythms in constant dim light. F Biol Rhythms 1997;12:467-77.

19 Hatonen T, Alila A, Laakso ML. Exogenous melatonin fails to counteract the light-induced phase delay of human melatonin rhythms. Brain Res 1996;710:125-30.

20 Cagnacci A, Soldani R, Yen SSC. Contemporaneous melatonin administration modifies the circadian response to nocturnal bright light stimuli. $A m \mathcal{F}$ Physiol 1997;41:R482-6.

\title{
Melatonin: a position statement of the British Olympic Association
}

\author{
Thomas Reilly, Ron Maughan, Richard Budgett
}

Exercise Physiology Steering Group of the British Olympic Association T Reilly

Nutrition Steering Group of the British Olympic Association R Maughan

\section{British Olympic Medical Centre R Budgett}

Correspondence to: Professor T Reilly, Research Institute for Sport and Exercise Sciences, Liverpool John Moores University, Byrom Street, Liverpool L3 3AF, United Kingdom.

Accepted for publication 15 January 1998
Melatonin is produced naturally by the pineal gland, within the base of the hypothalamus. There is a circadian rhythm in melatonin secretion starting at about 2100 hours and ending at about 0800 hours. This period is lengthened slightly in the winter and shortened a little during summer because the rhythm is linked to the hours of darkness, being inhibited by light (natural daylight or bright artificial light).

Melatonin is a hormone that provides a time signal for the normal circadian and annual rhythms in humans. It has: $(a)$ a lowering effect on body temperature, possibly through its actions on the peripheral blood vessels; $(b)$ a sleep inducing hypnotic effect; and $(c)$ in some circumstances an ability to shift the timing of the internal body clock forwards or backwards (its so called phase-response curve), depending on the timing of the dose.

Administration of melatonin leads to a rapid reduction in alertness and promotion of fatigue. Performance of mentally demanding tasks (requiring attention, vigilance, detection of errors) is impaired for five or six hours after ingestion. Melatonin is an antioxidant and hence used to combat free radicals. The main claims for its use have been as a hypnotic to aid sleep and as an antidote to jet lag.

Melatonin is not licensed for use in the United Kingdom and, like sleeping pills, is available only on prescription. In the United Kingdom it is sometimes prescribed to alleviate symptoms associated with chronic fatigue syndrome and also to treat the malaise known as seasonal affective disorder. Both melatonin and benzodiazepines (sleeping pills) have unpredictable effects and may even slow down rather than accelerate adjustment to new time zones.

A number of athletes are known to take melatonin to try to shift their body clock favourably and overcome jet lag more quickly. The following points need to be taken into consideration.

(1) It is impossible to predict the effect on the body's rhythms unless the timing of the body clock is known. The athlete must have spent some days in a local time zone and slept normally. Once travelling, it becomes very difficult to predict the phase of the body clock, and melatonin may delay rather than accelerate adjustment to local time zones. If given before the trough of the circadian rhythm in body temperature, it will advance the body clock irrespective of local time. Conversely, if given after the trough of body temperature, the timing of the body clock will be delayed.

(2) Side effects are sleepiness, headache, nausea, fuzzy head, and giddiness. An allergic reaction may occur in 1 in 240 individuals.

(3) Preparations bought in the United States are not subjected to any proper control and not all are pure. There is a particular danger of buying a product containing cow pineal gland. Most commercially available tablets contain $3 \mathrm{mg}$ melatonin. The warnings on the bottle must be taken seriously.

(4) There is a 25 -fold difference in plasma levels in different individuals after a $5 \mathrm{mg}$ dose, so effects are difficult to predict.

If, despite the advice given by the British Olympic Association Medical Committee (see box), athletes contemplate taking melatonin, the following adverse effects and contraindications must be respected.

(1) No other drugs should be taken in association with melatonin apart from simple analgesics or the oral contraceptive. It is not advisable to combine melatonin with 
hypnotics such as benzodiazepines (sleeping pills).

(2) Individuals must be over 18 , healthy, and not pregnant.

(3) A history, or family history in a first degree relative, of psychiatric disorder is a contraindication (because of central effects and possible addiction).

(4) Migraine is a contraindication.

If athletes take melatonin acquired overseas, the following caveats should be underlined. They must be familiar with the effects on them as individuals and take all steps to minimise side effects or the chances of delaying adaptation to the local time zone. They must be in phase with the local time zone (resident for enough time to have a settled circadian rhythm, which may be up to two weeks).

An athlete deciding from his/her experience to take melatonin, should note the following.

(1) If travelling eastward, $2-5 \mathrm{mg}$ melatonin should be taken at 1700-1900 hours local

\section{Statement on sleeping pills and melatonin}

The British Olympic Association Medical Committee advises great caution in the use of drugs such as hypnotics (sleeping pills) or melatonin to help overcome jet-lag. It may be better to use other strategies as advised by the British Olympic Association Acclimatisation Advisory Group.

Melatonin is not licensed in the United Kingdom and sleeping pills are only available on prescription. These drugs have unpredictable effects, including prolonged drowsiness in some individuals and they may even slow adjustment to new time zones.

Only consider using sleeping pills or melatonin when travelling to compete if you have used them before and know the effect on you. It is essential that your team doctor and other sports science and medicine support staff, such as team psychologist, are closely involved with your strategy to overcome jet-lag as quickly as possible.

It is only during the period before adjustment has taken place that jet-lag is experienced. Once the body-clock is adjusted, jetlag does not return - until the next time you travel across time-zones (usually on the return journey home). time before departure and then at bedtime on arrival for four days. The predeparture treatment helps to adjust the body clock by a phase advance (as is required) rather than by a phase delay which might otherwise occur.

(2) If travelling westward, no melatonin should be taken before the flight, but, in the evening, 2-5 mg should be taken before 2300 hours at the destination (local time).

The above recommendations are based on the action of melatonin as a hypnotic rather than a chronobiotic, so avoiding daytime administration of the drug.

Bright light opposes the action of melatonin and therefore avoidance of light is just as important as exposure to bright light. On an eastward flight, bright light should be avoidedwhere possible after 2000 hours on the day of departure.

\section{Key points}

- Melatonin is a naturally occurring hormone which is stimulated by darkness and inhibited by light.

- Melatonin has hypnotic effects, thereby inducing fatigue and drowsiness. It also has the ability to adjust the body clock.

- The temperature lowering effect of melatonin seems to occur whenever it is administered. Utilisation of this effect is best achieved if it is taken in the early evening in the new local time.

- Use of melatonin to combat jet lag must take into account both its hypnotic action and its phase-response curve. Taken in the evening (body clock time), it advances the body clock; taken in the morning, it delays the body clock. It can have opposite effects to those desired if the timing of ingestion is not in harmony with both internal and external time signals.

- Melatonin is not licensed for use in the United Kingdom and the purity of stocks in drugstores overseas cannot be guaranteed.

- For these reasons in general, behavioural and natural means of adjusting the body clock (use of light, exercise, social activity, sleeping/ waking) are preferred to melatonin or sleeping pills for promoting body clock adjustment after long flights.

Contributions by all the members of both the Exercise Physiology Steering Group and the Nutrition Steering Group of the British Olympic Association are gratefully appreciated. 